\title{
Las Auctoritates et uersus Strelabi del manuscrito 981 de la Abadía de Montserrat
}

\author{
Marta Cruz TrujILlo \\ Universidad Complutense de Madrid \\ martacruz@estumail.ucm.es
}

Recibido: 11 de enero de 2011

Aceptado: 17 de febrero de 2011

\section{RESUMEN}

El manuscrito 981 de la Abadía de Montserrat contiene un florilegio latino del siglo XV con una selección única de autores y obras, entre las que se encuentra la sección titulada Auctoritates et uersus Strelabi. Bajo este título se presentan extractos de la obra Monita ad Astralabium o Carmen ad Astralabium de Pedro Abelardo, pero también se recogen pasajes de las Fabulae de Gualtero el Inglés. En el trabajo se edita el texto de las Auctoritates et uersus Strelabi y se estudian las fuentes utilizadas así como la finalidad de esta sección en el conjunto del florilegio.

Palabras clave: Florilegio. Pedro Abelardo. Monita ad Astrolabium. Gualtero el Inglés. Anónimo de Nevelet. Fabulae.

Cruz Trujillo, M., «Las Auctoritates et uersus Strelabi del manuscrito 981 de la Abadía de Montserrat», Cuad. Fil. Clás. Estud. Lat. 31.1 (2011) 97-110.

\section{The Auctoritates et uersus Strelabi in the manuscript 981 of the Montserrat Abbey}

\begin{abstract}
The 981 manuscript of the Montserrat Abbey is a Latin florilegium dating from the 15th century with an unique and outstanding range of authors and works, among which is the entitled section Auctoritates et uersus Strelabi. This section not only contains extracts of the Pedro Abelardo's Monita ad Astralabium or Carmen ad Astralabium literary work, but also offers passages of the Fabulae of Walter of England holding the same title. Furthermore, the text of Auctoritates et uersus Strelabi is edited in this article, and the literary sources will be studied as well as the purpose of this section in the florilegium.
\end{abstract}

Keywords: Florilegium. Peter Abelard. Monita ad Astrolabium. Walter of England. Anonymus Neveleti. Fabulae.

Cruz Trujillo, M., «The Auctoritates et uersus Strelabi in the manuscript 981 of the Montserrat Abbey», Cuad. Fil. Clás. Estud. Lat. 31.1 (2011) 97-110.

SuMARIo 1. Introducción: el manuscrito 981 de la Abadía de Montserrat. 2. Las Auctoritates et uersus Strelabi. 3. Edición de los extractos de Monita ad Astralabium del manuscrito 981 de la Abadía de Montserrat. 3.1. Consideraciones previas a la edición. 3.2. Edición del texto. 4. Conclusiones. 5. Referencias bibliográficas. 


\section{INTRODUCCIÓN: EL MANUSCRITO 981 DE LA ABADÍA DE MONTSERRAT}

El manuscrito 981 de la Abadía de Montserrat es un florilegio latino medieval de la segunda mitad del siglo XV que ofrece una selección única de autores y obras ${ }^{1}$. Está escrito en letra gótica cursiva en papel, consta de 84 folios y sus medidas (21.6 x $14.7 \mathrm{~cm}$.; 15.7 x $9 \mathrm{~cm}$.) llevan a pensar que se trataba de un libro de bolsillo de uso particular, aunque las dimensiones han sido reducidas al haberse recortado sus márgenes (Villar Rubio 1995, p.222). Además, la cuidada y elegante caligrafía y la decoración de iniciales y capitales indica que su coste, como obra material, tuvo que ser elevado.

En opinión de Milagros Villar (1995, p.224), «por el tipo de dictámenes y sentencias recogidas en este volumen, debió pertenecer a algún "dictador" de la cancillería de la Corona de Aragón»; posteriormente perteneció al Marqués de Barberá. Si bien la mayor parte del códice está escrito en latín, el florilegio presenta una de sus secciones con el título escrito en catalán: Auctoritats de alguns actors. Además, hay unas anotaciones en la primera hoja de guarda ${ }^{2}$ que concuerdan perfectamente con el ambiente cancilleresco en el que fue confeccionado el florilegio, ya que son sentencias morales y civiles presentadas como una serie de preceptos para los consejeros de la Cancillería de Aragón. Están divididas en tres partes: la primera lleva por título Duodecim sunt uerba et facta per que perit hodie totus mundus y es la única de las tres que presenta sentencias escritas en latín. La segunda parte y la tercera están en catalán; la primera de estas dos aparece introducida por el texto: Les coses davall escrites son comunes a consell, e som $V$, y recoge las siguientes sentencias:

Primo: esperansa sola en Déu.

Secundo: diligència en los affers.

Tercio: secret en los consells.

Quarto: concòrdia entre nos.

Quinto: amar la cosa pública.

La tercera y última parte señala: Les VIII davall escrites pertanyen a sol càrrech dels consellers. Estas sentencias catalanas así como el título de la sección Auctoritats de alguns actors respaldan el origen catalán del códice.

La selección total de autores que extracta este florilegio no se encuentra en ningún otro manuscrito, al menos hasta el momento, por lo que se trata de un unicum. En esta selección se puede encontrar autores clásicos, tanto griegos como latinos, y autores más modernos, si atendemos a la fecha en la que se confeccionó el florilegio.

\footnotetext{
${ }^{1}$ Este estudio se enmarca dentro del proyecto I+D «Los florilegios latinos conservados en España IV», dirigido por la Dra. María José Muñoz (Ref.: FII2009-14489). Se puede consultar online el manuscrito en http://www.cervantesvirtual.com/obra-visor/recull-humanistic-llati-i-catala-manuscrit-0/html.

2 Aunque se trata de una hoja de guarda, sin embargo los catálogos la numeran como folio 1.
} 
La primera sección del manuscrito contiene excerpta de la obra de Petrarca De remediis utriusque fortunae y se titula Flores sumpte a magistro Petrarca laureato. Según ha demostrado $M^{a}$.J. Muñoz (e.p.), esta sección está tomada del manuscrito 19358 de la Biblioteca Nacional de Madrid. Ocupa 30 folios y presenta una división en el f.14v mediante la cual separa los dos libros del De remediis, dedicado el primero a la Próspera Fortuna y el segundo a la Adversa Fortuna.

El siguiente apartado (ff.30r-40v) recoge bajo el título Auctoritates et sume Titi Livii scilicet prime deche breves sentencias sacadas de la primera década de la obra de Tito Livio y organizadas siguiendo el orden de los diez primeros libros.

En la tercera sección el compilador del florilegio ofrece unas Auctoritats de alguns actors (Cruz Trujillo, e.p.). Es la única que presenta el título en lengua catalana, motivo por el cual se considera, como ya se ha mencionado más arriba, que este manuscrito es de origen catalán. Los autores recogidos en esta sección, si atendemos a las atribuciones dadas por el compilador, son: Platón, Valerio Máximo, Salustio, Pericles, Anaxágoras, Catón, Jenofonte, César Augusto, San Gregorio y Séneca, el autor más extractado en este apartado. Sin embargo, las sentencias aquí atribuidas a Pericles, Anaxágoras, Catón y Jenofonte son extractos de la obra Epistolae de rebus familiaribus en los que Petrarca cita a dichos autores, según he podido comprobar al analizar dichos extractos. Además, algunas de las sentencias atribuidas a Séneca y Platón y la de César Augusto pertenecen al De vita solitaria de Petrarca. No obstante, no es la única presencia oculta de esta sección, sino que entre estas sentencias también se encuentra extractado el autor Boncompagno da Signa y su obra Rhetorica nouissima. En esta ocasión dichos extractos se atribuyen a Valerio Máximo. Es muy posible que esta sección, Auctoritats de alguns actors, sea una selección de extractos novedosa y no copiada de ninguna otra colección medieval, sino confeccionada por el propio compilador del florilegio. Las presencias ocultas de Boncompagno y Petrarca y el título exclusivo en lengua catalana de la recopilación subrayan esta hipótesis.

Tras esta sección el compilador ofrece sentencias del Candelabrum de Bene de Florencia (ff.50v-55r), escrito alrededor de $1220^{3}$ y compuesto por ocho libros. En esta obra, también conocida como Candelabrum rethorici, Bene trata cuestiones generales de gramática, retórica y oratoria, ya que se trata de un manual de ars dictaminis. El modelo utilizado para realizar esta selección fue, según he podido comprobar (Cruz Trujillo 2011, e.p.), el ms. 9010 de la Biblioteca Nacional de Madrid, que presenta el Candelabrum completo. En el ms. 981 se han seleccionado solamente las máximas que Bene de Florencia ofrece al final de su obra como una serie de preceptos o consejos de carácter moral para el lector. La presencia de los extractos del Candelabrum en el florilegio es una muestra de la vigencia y el interés por el ars dictaminis en territorio hispano en el $\mathrm{s}$. XV, especialmente en el círculo dictatorial de la Cancillería de Aragón ${ }^{4}$.

${ }^{3}$ La cuestión de la cronología del Candelabrum de Bene de Florencia ha sido un tema muy discutido. $C f$. para un tratamiento extenso de dicha cuestión A. Gaudenzi (1895, pp.150-162).

${ }^{4}$ El manuscrito 9010 fue estudiado y anotado por cancilleres catalanes (Rico 1983, p.278); $c f$. sobre el aumento en el interés por el dictamen en las postrimerías del siglo XIV en el ambiente cancilleresco de Cataluña y Valencia F. Rico (1983, passim). 
Tras las sentencias de Bene de Florencia el compilador ofrece una colección de auctoritates titulada Auctoritates auctorum infra designatorum in marginibus (ff.55v-63v) con excerpta de Séneca, Aristóteles, Santo Tomás, San Gregorio, Valerio Máximo... En esta ocasión los nombres de los autores extractados aparecen escritos en los márgenes y en color rojo. Probablemente esta selección de máximas fue copiada de una colección de sentencias distinta e independiente de la de Auctoritats de alguns actors ${ }^{5}$. E igualmente a una recopilación distinta corresponden los Dicta philosophorum (ff.64r-70v) de diversos autores antiguos: Pitágoras, Sócrates, Platón, San Agustín, Aristóteles... ${ }^{6}$.

La siguiente sección bajo el título Auctoritates et sume parui Vrbani (ff.71r-74v) recoge los extractos de la obra de Daniel de Beccles Parvus Cato, también llamada Vrbanus Paruus y más conocida como Facetus. Esta obra, que ofrece una serie de consejos o reglas para saber comportarse en toda situación de la vida cotidiana, debe entenderse como una introducción a los Disticha de Catón. De hecho, se ha transmitido en numerosas ocasiones dentro de los llamados Libri Catoniani, al igual que los Monita ad Astralabium de Abelardo y las Fabulae de Gualtero el Inglés, obras también extractadas en este florilegio. Del Facetus de Daniel de Beccles existen dos versiones, una de ellas en hexámetros, que es la que se ha extractado en el florilegio. En esta ocasión los extractos se encuentran introducidos por calderones de color rojo.

El apartado que sigue a las sentencias de Daniel de Beccles se titula Auctoritates et uersus Strelabi (Lámina I: f.74v) y presenta en los folios $74 \mathrm{v}-76 \mathrm{v}$, al menos en apariencia, una selección de la obra Monita ad Astralabium o Carmen ad Astralabium de Pedro Abelardo, obra que contenía una serie de consejos de carácter moral; pero, como veremos más adelante, incluye también extractos de las Fabulae de Gualtero el Inglés.

Tras la sección de Pedro Abelardo el compilador ofrece siete refranes con rima interna y de carácter misógino (f.77r), y a continuación un nuevo apartado con los extractos de la obra de Ptolomeo bajo el título Hec sunt de doctrinis et sapienciis Ptolomei magni Strologi. Esta sección ocupa un folio y medio (ff.77r-78r) y su decoración, tal y como ocurre al final de numerosos manuscritos, parece estar sin acabar.

La compilación se cierra con una Doctrina beati Bernardi abbatis Clare uallis que dicitur Speculum anime super emendationem uite (ff.78v-80v), dedicada a los dicta de San Bernardo de Claraval. Al igual que en el caso de los extractos de Ptolomeo es un apartado muy descuidado en su presentación. Algunos extractos han sido anotados en los márgenes, y el medio utilizado para separar las sentencias es una serie de líneas verticales, en lugar de los calderones y rúbricas utilizados a lo largo de todo el florilegio.

Por último, los folios 81 y 82 están en blanco y el f. 83 presenta unas anotaciones y esquemas muy parecidos a los ofrecidos en la hoja de guarda, aunque en esta ocasión parece presentar un pequeño título, De memoria. Estas anotaciones vienen a corresponderse con las presentadas en la página de guarda inicial.

\footnotetext{
${ }^{5}$ El gusto por los libri auctoritatum y su utilización en el humanismo catalán es puesto de manifiesto también por F. Rico (1983, passim).

${ }^{6}$ Una tarea a realizar, y de la que me ocuparé en un futuro, es localizar los modelos de las distintas colecciones que reúnen auctoritates y dicta de diversos autores.
} 
Es característico de los florilegios otorgar a los extractos seleccionados una unidad y subordinarlos a una finalidad superior de acuerdo con los intereses del compilador (Aldama Roy - Muñoz Jiménez 2005, pp.217-218). En el caso del florilegio de Montserrat, parece haber sido compuesto con la intención de servir a su poseedor para formarse en el civismo y la moralidad, como si de un libro de buenas maneras se tratase. En este sentido, las diversas secciones han sido especialmente seleccionadas e incluso el texto original es en muchas ocasiones modificado. No se debe obviar que, como ya señaló $M^{\mathrm{a}}$.J. Muñoz (2004, p.124), al tratar sobre las características del florilegio:

nos encontramos con una obra que encierra en sí misma una doble condición: la de ser una producción original y nueva, por una parte, y, por otra, la de ser copia y testimonio de las distintas obras que componen la selección.

\section{LAS AUCTORITATES ET VERSUS STRELABI}

La sección en la que quiero centrar mi atención es la titulada Auctoritates et uersus Strelabi, un título que esconde los extractos de la obra Monita ad Astralabium (o Carmen ad Astralabium) de Pedro Abelardo, filósofo y teólogo francés que vivió a finales del s. XI y la primera mitad del XII. Además de dedicarse al estudio de la Lógica y otras materias, Pedro Abelardo se dedicó también a la enseñanza y a la música. Conoció a Eloísa, sobrina del canónigo de la Catedral de París, al serle encomendada su educación; mantuvieron una relación en secreto hasta que, al dar Eloísa a luz al hijo de ambos, Astrolabio, estalló el escándalo. Como consecuencia de una serie de intrigas, Abelardo fue castrado por orden del padre de Eloísa, y los dos amantes acabaron entrando en un convento. Más tarde Abelardo se enfrentó por motivos teológicos a Bernardo de Claraval, que curiosamente también se encuentra extractado en este florilegio.

Es precisamente al hijo que tuvo con Eloísa, Astrolabio, al que le dedicó la obra extractada en el manuscrito 981, tal y como nos indica el título de la misma. Esta obra de Abelardo contiene, en dísticos elegíacos, una serie de reglas de educación en sentido moral. De los Monita ad Astralabium de Abelardo existen tres versiones, dos de 445 versos y 461 versos y una más larga y completa de 1042 versos $^{7}$.

En las Auctoritates et uersus Strelabi del códice de Montserrat se ofrecen 41 extractos en los folios 74v-76v divididos en dos bloques, pues el extracto XII se presenta introducido por una inicial mayúscula de mayor tamaño que las capitales y decorada en rojo y azul (Lámina II: f.75r).

Una vez realizada la colación del texto, hemos podido establecer que las sentencias de este florilegio han sido extraídas de la versión más larga, ya que aparecen dísticos como los del extracto XII que no encontramos en las dos versiones más breves:

\footnotetext{
${ }^{7}$ Las versiones abreviadas no siguen la misma disposición que la versión más completa.
} 


\begin{abstract}
XII. Largus quisque datis, acceptis gaudet auarus;
Nec, si peniteat post data, largus erit.

Gaudet cum dederit largus, tristatur auarus;

rapta tamen cupido quam dona credo magis.

Qui leuiter spondet non multum soluere curat;

uir uerax minus hoc, sed magis illud agit.

Quam non precedit meritum promissio donum est:

cum hoc promiseris debitor efficeris.
\end{abstract}

Ahora bien, a la obra de Abelardo sólo pertenecen las 19 primeras sentencias, ya que las demás están extractadas de las Fabulae de Gualtero el Inglés; no obstante, el compilador no señala dicha autoría, sino que presenta los extractos de las Fabulae como si se trataran de más sentencias de Abelardo.

Gualtero el Inglés (Gualterius Anglicus) es frecuentemente identificado como Gualtero de Palermo (Gualterius Panormitanus) o como el Anónimo de Nevelet ${ }^{8}$. Fue Capellán de Enrique II de Inglaterra y se le encargó la educación de Guillermo II, futuro esposo de la hija de aquél, Juana. Para recompensarle por su labor y por el afecto que le debió de profesar, Guillermo le nombró arzobispo de Palermo y primado del reino de Sicilia. Es muy probable que en este contexto de educación del príncipe compusiese Gualtero sus Fábulas en dísticos elegíacos.

Durante la Edad Media no se conoció a Esopo ni a Fedro de forma directa, sino que se utilizaron diversas colecciones en verso o en prosa (Martín García 1996). Una de las más usadas fue la de Romulus o Aesopus latinus, que recoge versiones latinas en prosa de Fedro y de otras colecciones de fábulas griegas. Precisamente de las fábulas de Romulus, en concreto de los tres primeros libros, tomó Gualtero el Inglés sus 64 fábulas, las cuales se hallan extractadas en el florilegio, y las corrigió y adaptó al metro elegíaco.

Por su parte, en el manuscrito de Montserrat los extractos pertenecen siempre a los dos últimos versos de las fábulas, es decir, que lo que se extracta es la morale$\mathrm{ja}^{9}$. Por tanto, interesa la sentencia universal, más general, para complementar las reglas prácticas que ofrece la obra de Abelardo extractada en la primera parte de la sección.

Por otro lado, si atendemos al conjunto del florilegio, creemos que hay que poner en relación la presencia de los extractos de Abelardo y de las Fabulae con los de la sección Auctoritates et sume parui Vrbani, que recoge sentencias del Facetus de Daniel de Beccles y precede al apartado Auctoritates et uersus Strelabi. Como ya se ha expuesto más arriba, el Facetus también contiene una serie de 'reglas de educación y buenas maneras' y proporciona consejos y normas para el desarrollo de la vida cotidiana. Abelardo y el Esopo de Gualtero tienen también ese sentido práctico y educativo, por lo que debe entenderse una finalidad unitaria en los extractos de las tres

\footnotetext{
${ }^{8}$ Cf. sobre la identidad del autor de las Fábulas en dísticos elegíacos, L. Hervieux (1970, pp.472-495); C. Roccaro (1998) y Häuptli (2005).

${ }^{9}$ El mismo procedimiento de selección se utiliza en el manuscrito 150 del Archivo Capitular de Córdoba (Fernández de la Cuesta 2008, p.263).
} 
obras, didáctica y edificante. Hay que tener en cuenta que tanto el Facetus como las Fabulae de Gualtero formaron parte de los Libri Catoniani, los cuales recogían textos que se utilizaban en la enseñanza medieval. El canon de autores de los Libri Catoniani varió a lo largo de los siglos, siendo recogida la obra de Gualtero el Inglés desde fines del s.XII y el Facetus a partir del s.XIII (Fernández de la Cuesta 2008, pp.112-113). Se acentúa por tanto aún más el carácter práctico y pedagógico de estas secciones y se puede conjeturar que en el archivo de la Cancillería de Aragón existía un Liber Catonianus perteneciente al último estado de la tradición canónica, que ya incluiría obras como el Facetus y las Fabulae de Gualtero el Inglés.

\section{EDICIÓN DE LOS EXTRACTOS DE MONITA AD ASTRALABIVM DEL MANUSCRITO 981 DE LA ABADÍA DE MONTSERRAT.}

\subsection{CONSIDERACIONES PREVIAS A LA EDICIÓN.}

El ms. 981 es un florilegio latino medieval y, como tal, a la hora de elaborar la edición deben respetarse no sólo la estructura y organización que dispuso el compilador del mismo, sino también las características propias del estado evolutivo en el que se encontraba su lengua. Así lo señala $\mathrm{M}^{\mathrm{a}}$.J. Muñoz (2004, p.127):

El texto que ha de ser editado ha de reflejar el estado de lengua propio del compilador que hizo la selección, es decir, que, por más que sean textos de autores clásicos los que compongan el florilegio, no habrá que 'restituir a lo clásico'. [...] Por otra parte, en la organización de los extractos, pasajes y unidades textuales se atenderá a la presentada por el exceptor $[\ldots]$.

Además, desarrollamos en nuestra edición un aparato de fuentes que permite identificar la procedencia de los extractos y un aparato intertextual en el que se reflejan las variantes del florilegio frente al texto completo de las obras seleccionadas (Muñoz 2004, p.131). En el caso de los Monita ad Astralabium de Abelardo utilizamos la edición de Graziella Ballanti y para los extractos de Gualtero el Inglés, la edición de sus Fabulae a cargo de Léopold Hervieux.

\section{Conspectus siglorum}

fl.: ms. 981 de la Abadía de Montserrat.

m: G. Ballanti (ed.), Pedro Abelardo, Insegnamenti al figlio. Commento, traduzione e testo latino, Roma 1993.

$h$ : L. Hervieux(ed.), Les fabulistes latins. Depuis le siècle d'Auguste jusqu'à la fin du moyen âge, vol. II, Hildesheim 1970 (reproducción de la edición de París 1893 y 1894), pp.316-51. 


\subsection{EDICIÓN DE LOS EXTRACTOS EN EL MANUSCRITO 981 DE LA ABADÍA DE MONTSERRAT.}

\section{Auctoritates et uersus Strelabi}

I. Cum bene quis moritur persoluit debita cuncta, nil quod agat superest, plus nichil exhigitur (sic).

$\mathrm{Ne}$ moriens aliis tua distribuenda relinquas: nullus erit quam tu plus tibi propicius.

Nemo tuos melius tua dispensabit in usus.

II. Dum poteris prodesse tuis, properare memento.

Vita breuis fortuna leuis tibi sit documento.

III. Cum modicum membrum sit lingua, est maximus ignis,

Non tot per gladium quot periere per hanc.

Preualet in lingua qui non est fortis in armis;

nullus in hac pugna plus meretrice potest.

Ex hoc precipue distant ignauus et audax, quod factis iste preualet, ille minus.

Si lingue bellum quam armorum forcior esset, Tresites Troie maior Achille foret.

IV. Ne libertatem uendas, fuge dona potentum:

Emptum te credas hec ubi sustulerit.

V. Quas multi multo conquirunt tempore patres

stultus eas modico dissipat unus opes.

VI. Sicut amat sapiens hominem qui corrigit ipsum,

sic hodit qui se corripit incipiens.

Opprobriis propriis aurem dat nemo libenter, nec te nec quemquam talia scire uolet.

VII. Si sit amica senex, donis conducat amicum, suppleat ex donis quod caret ex facie.

VIII. Vt largus largo sic sis, et auarus auaro; qualis hic est aliis sis quoque talis ei.

IX. Religio iuuenis leuis est impulsio mentis et tanquam torrens impetuosus aque.

$\mathrm{X}$. Velocem quemcumque pigrum facit escha parata;

Tunch (sic) si miseris hunc, desinit esse piger.

XI. Quid, cui des uideas, ubi, quando, quomodo, quare, ni grates pariter donaque perdere uis.

I: Abel. Mon. 613-7 | II: a.i. | III: Abel. Mon. 235-42 | IV: Abel. Mon. 273-4 | V: Abel. Mon. 742-3 | VI: Abel. Mon. 524-562 | VII: Abel. Mon. 323-4 | VIII: Abel. Mon. 781-2 | IX: Abel. Mon. 663-4 | X: Abel. Mon. 433-4 | XI: Abel. Mon. 395-6

1 cum $f l:$ dum $m 9$ non $f l .:$ nec $m \| \mathbf{1 1}$ potest $f l .:$ valet $m \| \mathbf{1 3}$ minus $f l .:$ minis $m \| \mathbf{1 5}$ Tresites $f l .:$ Thersites $m \| \mathbf{1 8}$ patres $f l$ : docti $m \| \mathbf{2 0}$ ipsum $f l$ : illum $m \| \mathbf{2 1}$ corripit $f l .:$ corrigit $m \mid$ incipiens $f l .:$ insipiens $m \| \mathbf{2 2}$ propriis aurem $f l .:$ aurem propriis $m \| \mathbf{2 7}$ et avarus $f l .:$ avarus $m \| \mathbf{3 1}$ tunch $f l:$ huc $m$ 
XII. Largus quisque datis, acceptis gaudet auarus;

Nec, si peniteat post data, largus erit.

Gaudet cum dederit largus, tristatur auarus;

rapta tamen cupido quam dona credo magis.

Qui leuiter spondet non multum soluere curat;

uir uerax minus hoc, sed magis illud agit.

Quam non precedit meritum promissio donum est:

cum hoc promiseris debitor efficeris.

XIII. Si taceat stultus, sapiens reputabitur esse;

nil sic ut sermo prodere corda solet.

XIV. Infames fugiat tua conuersacio semper

et socio gaude te meliore frui.

$\mathrm{XV}$. Scit sibi, non aliis, qui nescit scita docere;

tamquam nil si scierit talis habendus erit.

XVI. Si non subuenias donec te exoret amicus

quod dare te credis uendere crede magis.

XVII. Antiquum retine, quem sis expertum, amicum,

nec similem credas, si sapis, esse nouum.

XVIII. Qui fugit ypocrite nomen pecando patenter

duppliciter reus est, qui docet hoc et agit.

XIX. Verborum leuitas morum fert pondus honestum

et nucleum celat arida testa bonum.

$X X$. Sic nocet innocuo nocuus causamque nocendi

inuenit. Hii regnant qualibet urbe lupi.

XXI. Sic pereant qui se prodesse fatentur;

Discat in actorem pena redire suum.

XXII. Sepe fidem falso mendicat in hercia ( sic) teste;

Sepe dolet pietas criminis arte rapi.

XXIII. Non igitur debent pro uariis certa relinqui;

non sua si quis auet, mox caret ipse suis.

XXIV. Nil prodest prodesse: mens praua malorum

inmemor accepti non timet esse boni.

XXV. Non satis est tutum mollitis credere uerbis;

ex hoc melle solet pestis amara sequi.

XII: Abel. Mon. 364-405, 415-8 | XIII: Abel. Mon. 825-6 | XIV: Abel. Mon. 725-6 |XV: Abel. Mon. 801-2 |XVI: Abel. Mon. 139-40 | XVII: Abel. Mon. 1-190 | XVIII: Abel. Mon. 307-8 |XIX: Gualt. Fab. pról. 11-2 | XX: Gualt. Fab. II 15-6 | XXI: Gualt. Fab. III 15-6 | XXII: Gualt. Fab. IV 9-10 | XXIII: Gualt. Fab. V 5-6 | XXIV: Gualt. Fab. VIII 7-8 |XXV: Gualt. Fab. IX, 11-2

|| $\mathbf{3 7}$ dona $f l:$ : data $m \| \mathbf{4 0}$ meritum promissio $f l .:$ promissio $m \mid$ donum $f l .:$ gratia donum $m \|$ 41 hoc promiseris $f l$.. promiseris hoc $m \| \mathbf{4 7}$ si scierit $f l$.: scierit $m \| \mathbf{5 6}$ causamque $h$ : tamenque $f l$. $\| \mathbf{5 8}$ fatentur $f l$ : fatentur et obsunt $h \| \mathbf{6 0}$ in hercia $f l$ : inertia $h \| \mathbf{6 2}$ variis $f l .:$ vanis $h \| \mathbf{6 4}$ prodesse $f l .:$ prodesse malis $h \| \mathbf{6 6}$ mollitis $f l .:$ mellitis $h$ 
XXVI. Reddere gaudet homo nequam pro melle uenenum, pro fructu penam, pro pietate dolum.

XXVII. Pauperies si leta uenit, ditissima res est;

Tristior in mensas pauperat usus opes.

XXVIII. Hunc timeat casum, qui se non fulsit amico.

Nec dare uult felix, quam miser optat, opem.

XXIX. Displicet imprudens, quando placere putat;

quod natura negat, nemo feliciter audet.

XXX. Tu qui summa potes, ne despice parva potentem;

nam prodesse potest, si quis obesse nequit.

XXXI. Qui maculat uitam, mundas cur incolit aras?

Quem sua facta premunt, cur aliena leuat?

XXXII. Vtile consilium qui uitat, inutile summit;

qui nimis est tutus, recia iure subit.

XXXIII. Si quis habet quod habere decet, sit letus habendo;

Alterius non sit, qui suus esse potest.

XXXIV. Si quid agis, prudenter agas et respice finem;

fferre minora volo, ne grauiora feram.

XXXV. Tempore non omni, omnibus omnia credas:

qui misere credit, creditur esse miser.

XXXVI. Sepe minus faciunt homines, qui magna minantur:

sepe gerit nimios causa pusilla metus.

XXXVII. Quod tibi non faceres, aliis fecisse caueto,

Vulnera non facias, que nequis ipse pati.

XXXVIII. Audet in audacem timidus, fortique minatur

debilis, audendi cum uidet esse locum.

XXXIX. Sordibus imbuti nequeunt dimitere sordes;

Ffallere qui didicit, fallere semper auet.

XL. Quod non es, non esse uellis; quod es, esse fatere:

est male quod non est, qui negat esse quod est.

XLI. Tutorem retinere suum tutissima res est;

nam si tutor abiit, hostis obesse potest.

Expliciunt uersus Strelabii

XXVI: Gualt. Fab. X 11-2 |XXVII: Gualt. Fab. XII 31-2 | XXVIII: Gualt. Fab. XVI 11-2 | XXIX: Gualt. Fab. XVII 16-15 |XXX: Gualt. Fab. XVIII 23-4 |XXXI: Gualt. Fab. XIX 9-10 | XXXII: Gualt. Fab. XX 13-4 |XXXIII: Gualt. Fab. XXI 21-2 |XXXIV: Gualt. Fab. XXII 5-6 | XXXV: Gualt. Fab. XXIV 9-10 | XXXVI: Gualt. Fab. XXV 7-8 | XXXVII: Gualt. Fab. XXXIII,13-4 | XXXVIII: Gualt. Fab. XXXVI 9-10 | XXXIX: Gualt. Fab. XXXVIII 11-2 |XL: Gualt. Fab. XLII 15-6 | XLI: Gualt. Fab. LII 13-4

70 in mensas $f l .:$ immensas $h \quad \mid$ 74-75 $f l .:$ 75-74 $h \| \mathbf{7 4}$ quando $f l .:$ unde $h \| \mathbf{8 0}$ vitat $f l$.: spernit $h \| \mathbf{8 4}$ agis $f l .:$ agas $h \mid$ respice $f l .:$ respire $h \| \mathbf{8 6}$ omnibus $f l .:$ non omnibus $h$. $\mathbf{9 1}$ non $f l .:$ ne $h \mid$ nequis $f l:$ potes $h \| \mathbf{9 3}$ audendi $f l .:$ audenti $h \| \mathbf{9 6}$ esse fatere $f l .:$ fateris esse $h \| \mathbf{9 7}$ negat $f l .:$ sinit $h$ 


\section{CONCLUSIONES.}

El manuscrito 981 de Montserrat ofrece bajo el título de Auctoritates et uersus Strelabi extractos de la obra Monita ad Astralabium de Pedro Abelardo. De las tres versiones existentes de la obra, el texto del florilegio sigue la versión más larga, de 1042 versos, ya que ofrece dísticos que no se encuentran en las versiones abreviadas. Además, el compilador presenta en esta misma sección extractos de las Fabulae de Gualtero el Inglés, si bien no señala ningún cambio en la autoría. Esta presencia oculta no es un caso único en este florilegio, ya que también se ofrecen encubiertamente extractos de las Epistolae de rebus familiaribus y del De uita solitaria de Petrarca así como de la Rhetorica novissima de Boncompagno de Signa en la sección Auctoritats de alguns actors.

El contenido de las Auctoritates et uersus Strelabi debe ponerse en relación con la sección inmediatamente anterior, titulada Auctoritates et sume parui Vrbani y dedicada a los extractos del Facetus de Daniel de Beccles. La presencia de los extractos de los Monita ad Astralabium de Abelardo, del Facetus de Daniel de Beccles y de las Fabulae de Gualtero el Inglés en este florilegio parece corroborar la finalidad práctica y moral de la recopilación, sobre todo si tenemos en cuenta que lo único que se extracta de las fábulas es la moraleja final.

Precisamente es el carácter unitario lo que distingue a los florilegios de las antologías y otros tipos de colecciones de textos, ya que en él todos los extractos se encuentran fuera de su contexto inicial y subordinados a los intereses del compilador. Así lo señalan A.Ma Aldama y M $^{\mathrm{a}}$.J. Muñoz (2005, p.217):

[...] el florilegio no debe confundirse con las colecciones gnómicas o apotegmáticas de sententiae, aunque en ocasiones ofrece rasgos comunes con éstas y selecciona frases sentenciosas, ni con las grandes antologías poéticas o misceláneas de diversos géneros, pues aunque todas estas compilaciones seleccionan pasajes escogidos de diversos autores, en el florilegio los extractos se presentan organizados de forma unitaria y el compilador realiza una reescritura del texto original dándole una nueva finalidad.

Es muy probable que en la Cancillería de Aragón, donde fue confeccionado el florilegio, se conservara una colección de la versión más tardía de los Libri Catonia$n i$ que ofreciese el Facetus y las Fabulae de Gualtero el Inglés.

Por otro lado, el hecho de que el compilador atribuya extractos de unas obras o autores a autoridades distintas resulta una práctica bastante habitual en los florilegios latinos medievales. La presencia oculta de las Fabulae de Gualtero el Inglés demuestra, una vez más, la necesidad de estudiar los manuscritos de forma completa e individual y de localizar, en el caso de los florilegios, las fuentes de los extractos. Sólo así se conseguirá obtener una visión global y certera del panorama cultural de una época y lugar concretos; en este caso, el florilegio conservado en el manuscrito 981 de la Abadía de Montserrat es buen reflejo de los intereses de lectura en la Corona de Aragón en la primera mitad del siglo XV. 


\section{REFERENCIAS BIBLIOGRÁFICAS}

Aldama Roy, A.Ma . - Muñoz JimÉnez, Ma .J. (2005), «Los florilegios», en AnTón, B. - SigNES, J. ET ALII, Antiquae lectiones. La tradición greco-latina desde la Antigüedad hasta la Revolución Francesa, Valladolid, Cátedra, pp.217-223.

Ballanti, G. (1993), Pietro Abelardo. Insegnamenti al figlio, Commento, traduzione e testo latino, Roma, Armando Editore.

Cruz Trujillo, M. (2011), «El Candelabrum de Bene de Florencia en el manuscrito 981 de la Abadía de Montserrat», Boletín de la Real Academia de Buenas Letras 52, e.p.

Cruz Trujillo, M. (e.p.), «Auctoritats de alguns actors en el mss. 981 de la Abadía de Montserrat», Auctor et Auctoritas in Latinis Medii Aevi Litteris. Actas del VI Convegno dei International Medieval Latin Komitee (10-14 de noviembre de 2010).

FERnÁndez de la Cuesta, A. (2008), En la senda del 'Florilegium Gallicum'. Edición y estudio del florilegio del manuscrito Córdoba, Archivo Capitular 150, Lovaina la Nueva, Fédération Internationale des Instituts d'Études Médiévales (Coll. Textes et Études du Moyen Âge 45).

GAudEnZI, A. (1895), «Sulla cronologia delle opere dei dettattori Bolognesi di Boncompagno a Bene di Lucca», Bulletino dell'Instittuto Storico Italiano 14.85-162.

Hervieux, L. (1970), Les fabulistes latins. Depuis le siècle d'Auguste jusqu'à la fin du moyen âge, vol. I y II, Hildesheim - Nueva York, reproducción de la edición de París 1893 y 1894, Firmin-Didot.

HÄUptLI, B.W. (2005), «Walter von Palermo», en W.F. BAUTZ (ed.), Biographisch-Bibliographisches Kirchenlexikon, Band XXV, col.447-1453

MARTín GARCíA, F. (1996), Antología de fábulas esópicas en los autores castellanos (hasta el siglo XVIII), Cuenca, Servicio de Publicaciones de la Universidad de Castilla-La Mancha.

MuÑOZ JiMÉNEZ, Ma .J. (2004), «La edición de florilegios como ‘edición especial’», Exemplaria Classica 8, 123-133.

MuÑOZ JimÉNEZ, Mª.J. (e.p.), «Flores sumpte a magistro Patrarcha laureato en el manuscrito 981 de la Abadía de Montserrat», Auctor et Auctoritas in Latinis Medii Aevi Litteris. Atti del VI Convegno dei International Medieval Latin Komitee (10-14 de noviembre de 2010).

Olivar, A. (1977), Catàleg dels manuscrits de la Biblioteca del Monestir de Montserrat, Monasterio de Montserrat, Publicaciones de l'Abadía de Montserrat.

Rico Manrique, F. (1983), «Petrarca y el 'humanismo catalán'», Actes del sisè col·loqui internacional de llengua i literatura catalanes, Barcelona, Abadía de Montserrat, pp.257291.

Roccaro, C. (1998), «Sull'autore dell'Aesopus comunemente attribuito a Gualtiero Anglico», Pan: studi dell'Istituto di Filologia Latina «Giusto Monaco»15-16, 195-207.

Villar Rubio, M. (1995), Códices petrarquescos en España, Padua, Editrice Antenore. 


\section{LÁMINAS}

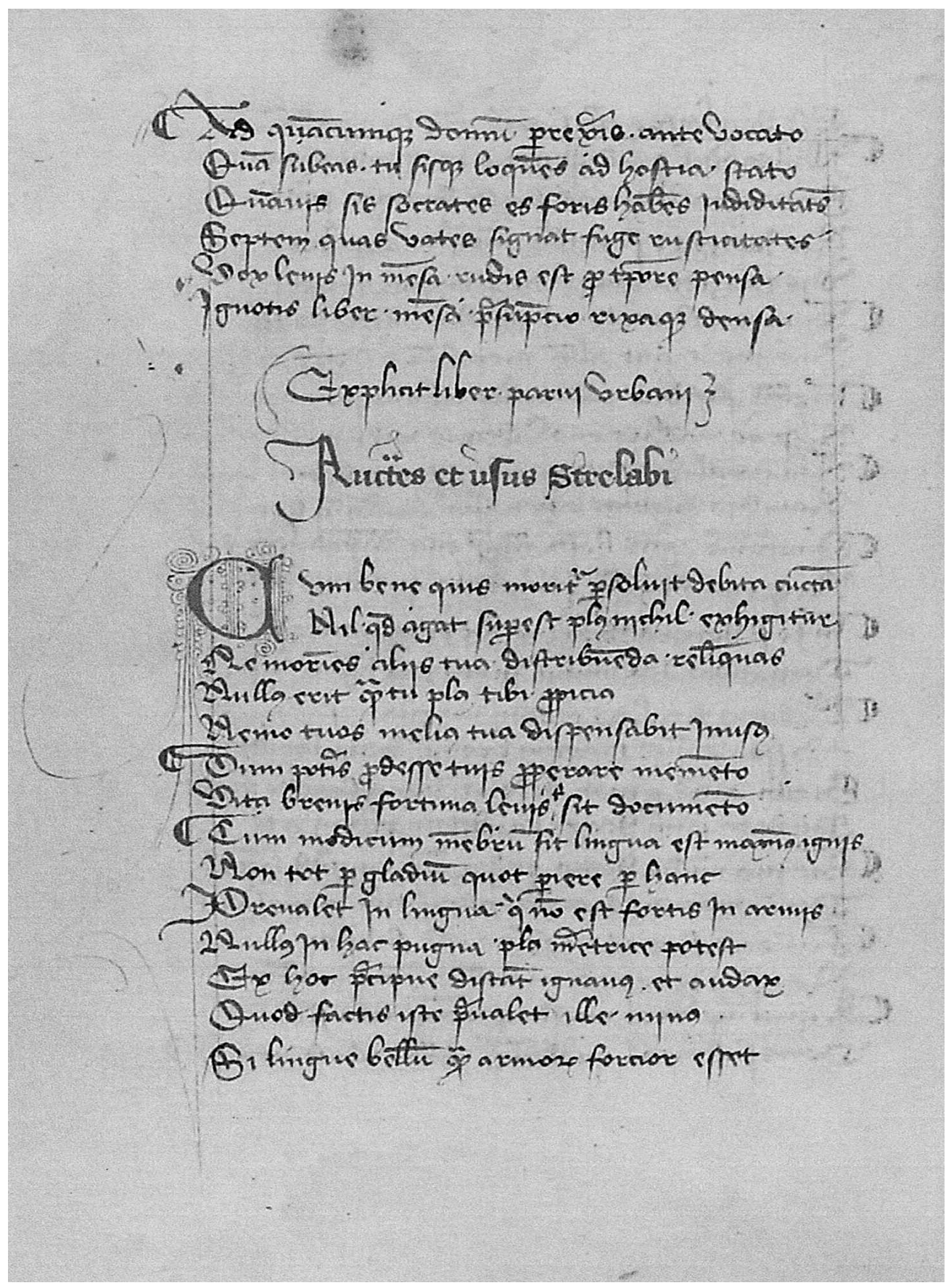

Lámina I: ms. 981 de la Abadía de Montserrat, f.74v 


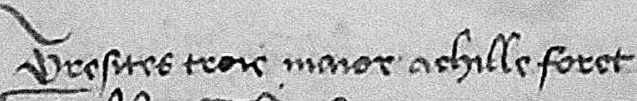

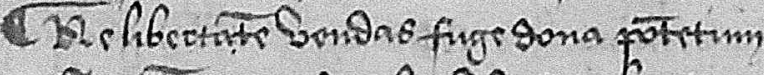

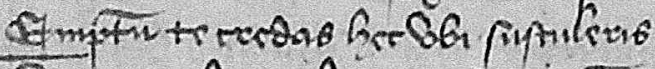

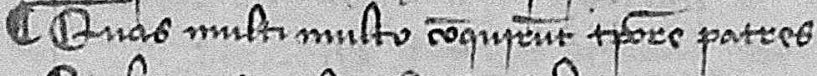
Btufto rate unosin sipprat thio apes

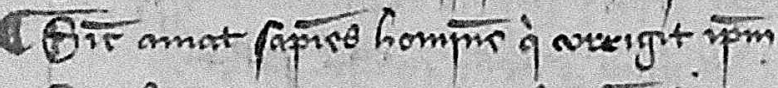

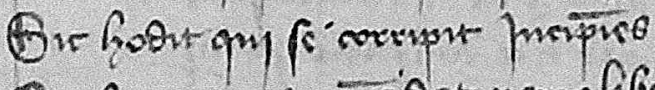

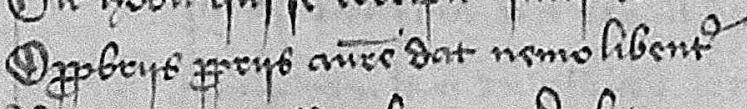
29etrimse ging talia pres bolst

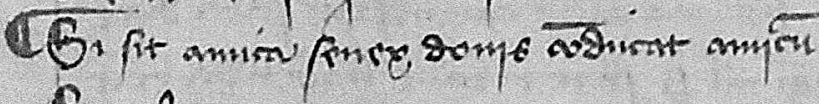

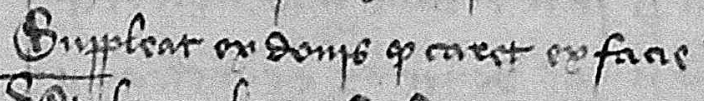

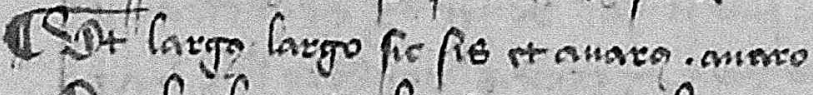

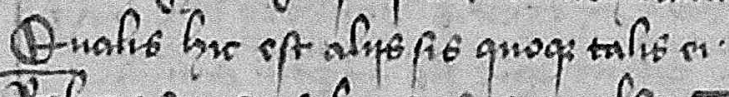

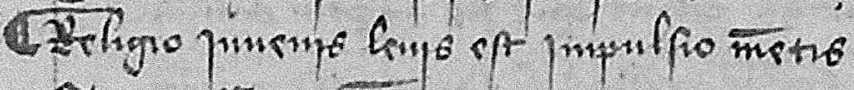
Et trong tovese pmpertiog aque (E)

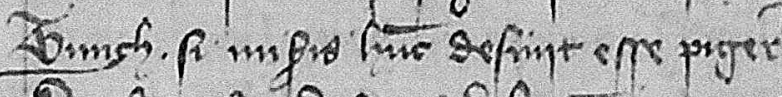

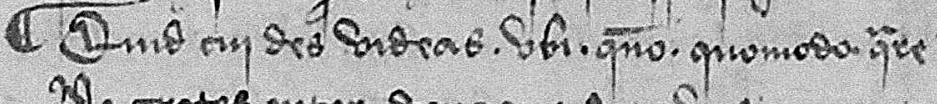

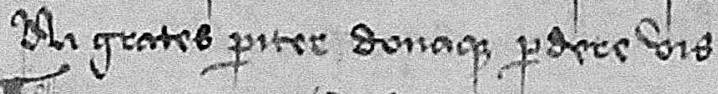

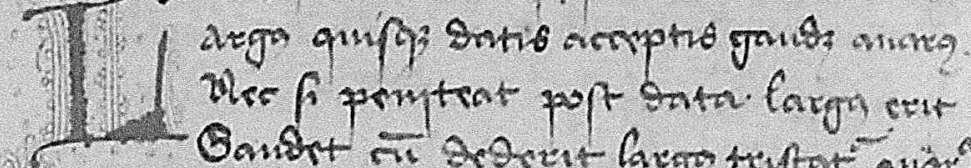

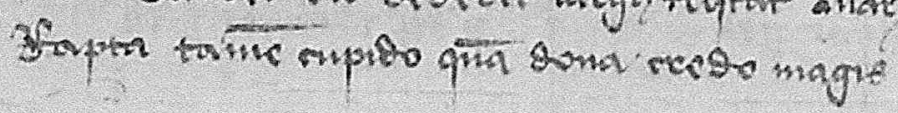

Lámina II: ms. 981 de la Abadía de Montserrat, f.75r 